\title{
Protein and Peptide Array Analysis of Autoimmune Disease
}

\author{
William H. Robinson ${ }^{1,2}$, Lawrence Steinman ${ }^{2}$, and Paul J. Utz ${ }^{1}$ \\ ${ }^{1}$ Division of Immunology and Rheumatology, Department of Medicine, and \\ ${ }^{2}$ Department of Neurology, Stanford University School of Medicine, Stanford, CA, USA
}

BioTechniques 33:S66-S69 (December 2002)

\section{ABSTRACT}

Molecular cloning, sequencing of the human genome, and other major advances in biomedical research have contributed substantially to our understanding of autoimmune disease. Nevertheless, to date, such advances have failed to reveal the etiology of or yield curative therapies for autoimmune disease. New approaches are needed. Proteomics, the large-scale study of expression and function of proteins that compose our tissues and mediate disease, represents a powerful and promising strategy. We developed protein and peptide arrays to profile autoantibody responses in autoimmune disease. Protein and peptide array analysis of autoimmune samples is revealing human and pathogen proteins involved in initiation and perpetuation of autoimmunity. Proteomic determination of autoantibody profiles can be utilized for diagnosis, prognostication, and guiding tolerizing therapy for autoimmune disease.

\section{INTRODUCTION}

Despite major advances in the biomedical sciences over the past three decades, including development of molecular biology techniques and sequencing of the human genome, autoimmune disease remains an enigma. Sequencing of the human genome revealed 30000 genes, estimated to encode 300000 polypeptides with distinct functional properties (32). These 300000 polypeptides, along with lipids and carbohydrates, compose our cells and tissues. Aberrant expression or function of one or more of these 300000 proteins can result in disease. The large-scale study of the expression and function of these proteins is termed proteomics (21), and proteomics offers the potential to identify and characterize functional aberrancies in proteins associated with autoimmunity and other diseases.

Autoimmune diseases, including rheumatoid arthritis (RA), multiple sclerosis (MS), and autoimmune diabetes (insulin-dependent diabetes mellitus [IDDM]), affect $3 \%$ of the population of the industrialized world (12). Despite significant research efforts, the etiology of and self-molecules targeted in the vast majority of autoimmune diseases remain poorly understood. For example, although Grave's disease and myasthenia gravis are known to target the thyroid hormone receptor and acetylcholine receptor, respectively, the targets of the autoimmune responses in inflammatory bowel disease and psoriasis are unknown. Autoimmunity is believed to arise in genetically predisposed individuals following exposure to environmental triggers (28). Proteomics technologies enable large-scale characterization of immune responses against pathogens and self-proteins and represent a powerful and promising strategy to identify pathogens and self-proteins involved in the initiation and progression of autoimmune disease (23). Knowledge of initiating pathogens could lead to more aggressive treatment of or vaccination against causative infectious agents. Knowledge of autoimmune self-protein targets would better enable development of antigen-specific tolerizing therapies $(24,25)$.

We have developed protein and peptide arrays, termed antigen arrays, to profile antibody responses against foreign and selfproteins in autoimmune diseases (23). This review will discuss the clinical applications of autoantibody profiling, including the use of such profiles in diagnosis, prognostication, and tailoring antigen-specific therapy.

\section{SHORTCOMINGS OF TRANSCRIPTIONAL PROFILING}

Several studies culled genes encoding proteins with pathophysiologic and therapeutic relevance from DNA microarray analysis of tissue derived from autoimmune lesions $(6,17)$. Nevertheless, mRNA transcipt profiling has important limitations and will likely prove insufficient to unravel the etiology of, and to develop next-generation therapeutics for, autoimmune disease. A growing number of studies suggest that relatively frequent discordance exists between mRNA and protein expression $(5,11,16$, 26). As outlined in Table 1 , in the study of autoimmune disease, RNA transcript profiling of certain genes is not informative with regard to net protein expression, protein function, and/or the specificity of antigen receptors. Proteomics, which is the direct study of the expression and function of proteins encoded by these RNA transcripts, circumvents many of the limitations of RNA transcript profiling and will likely provide critical insights into the mechanisms of autoimmune disease in the postgenomics era.

\section{AUTOANTIBODIES AS SURROGATES FOR THE SPECIFICITY OF T CELL-MEDIATED AUTOIMMUNE DISEASES}

Studying the specificity of autoimmune responses poses unique challenges. Autoreactive $\mathrm{T}$ and $\mathrm{B}$ lymphocytes, which are 
Table 1. Immunology Questions Not Adequately Addressed by Transcriptional Profiling

\begin{tabular}{|c|c|}
\hline Question & Examples \\
\hline \multirow[t]{2}{*}{ Protein expression } & $\begin{array}{l}\text { Expression of tumor necrosis factor (TNF)- } \alpha \text { protein, a driver of autoimmune tissue injury } \\
\text { in RA and MS, is regulated posttranscriptionally by } 3^{\prime} \text { untranslated region AU-rich } \\
\text { sequences (15). }\end{array}$ \\
\hline & $\begin{array}{l}\text { Polyadenylation signal sequences frequently regulate protein expression } \\
\text { posttranscriptionally (10). }\end{array}$ \\
\hline Alternatively spliced mRNAs & $\begin{array}{l}\text { Certain polypeptides with diametric functions, such as bcl- } x_{S} \text { and } b c l-x_{L}(13) \text {, arise from } \\
\text { alternative splicing of common RNA transcripts. }\end{array}$ \\
\hline Posttranslational modifications & $\begin{array}{l}\text { T and B lymphocyte activation are exquisitely regulated by phosphorylation and } \\
\text { dephosphorylation of the antigen receptor-associated signaling complexes. }\end{array}$ \\
\hline $\begin{array}{l}\text { Antigen receptor expression } \\
\text { and specificity }\end{array}$ & $\begin{array}{l}\text { Autoreactive } T \text { and } B \text { cells exist in heterogenous populations at frequencies of less than } \\
1: 10000(2,27) \text {, making transcriptional profiling of } T \text { cell receptor and autoantibody gene } \\
\text { usage uninformative. }\end{array}$ \\
\hline
\end{tabular}

the cells that provide specificity to autoimmune responses, exist in heterogenous populations at frequencies of less than 1:10000 lymphocytes $(2,27)$. Autoimmune responses are coordinated by autoreactive $\mathrm{CD}^{+}{ }^{+}$helper T cells; nevertheless, large-scale characterization of $\mathrm{T}$ cell specificity is currently not feasible. $\mathrm{T}$ cell proliferation assays require relatively large numbers of $T$ cells, thereby severely limiting the performance of multiplex assays. Production of major histocompatability complex (MHC)-peptide reagents (1), which enable direct identification of T cells of certain specificities using flow cytometry, is cumbersome, due to: $(i)$ the need to optimize the conditions to achieve proper folding of the MHC molecule with each peptide; and (ii) the instability of the resulting complexes. Thus, it is not currently possible to perform multiplex analysis of autoreactive $T$ cell specificities.

We hypothesize that, due to the reciprocal nature of $\mathrm{T}$ and $\mathrm{B}$ cell activation, the specificity of autoreactive $\mathrm{B}$ cell responses can be used as a surrogate for the specificity of the overall autoimmune response in $\mathrm{T}$ cell-mediated autoimmune diseases (Figure 1). B cells are professional antigen-presenting cells (APCs), and through their cell surface immunoglobulin they bind, endocytose, process, and present to $\mathrm{T}$ cells linear peptide fragments complexed to class II MHC on their cell surface. Because an individual $\mathrm{B}$ cell is only able to bind to antigens that its rearranged immunoglobulin recognizes, each B cell is only capable of activating and receiving activating signals from helper $T$ cells expressing a $T$ cell receptor specific for a peptide epitope derived from that macromolecular antigen (Figure 1). Autoreactive B cells secrete highaffinity autoantibodies that can be detected in serum, synovial fluid, and/or cerebral spinal fluid (CSF), thus greatly facilitating characterization of the specificity of autoreactive $\mathrm{B}$ cell responses.

\section{PROTEOMIC TECHNOLOGIES FOR AUTOANTIBODY PROFILING}

A variety of miniaturized proteomic technologies are amenable to profiling autoantibody responses. These include: $(i)$ arrays of living cells expressing transformed or transfected

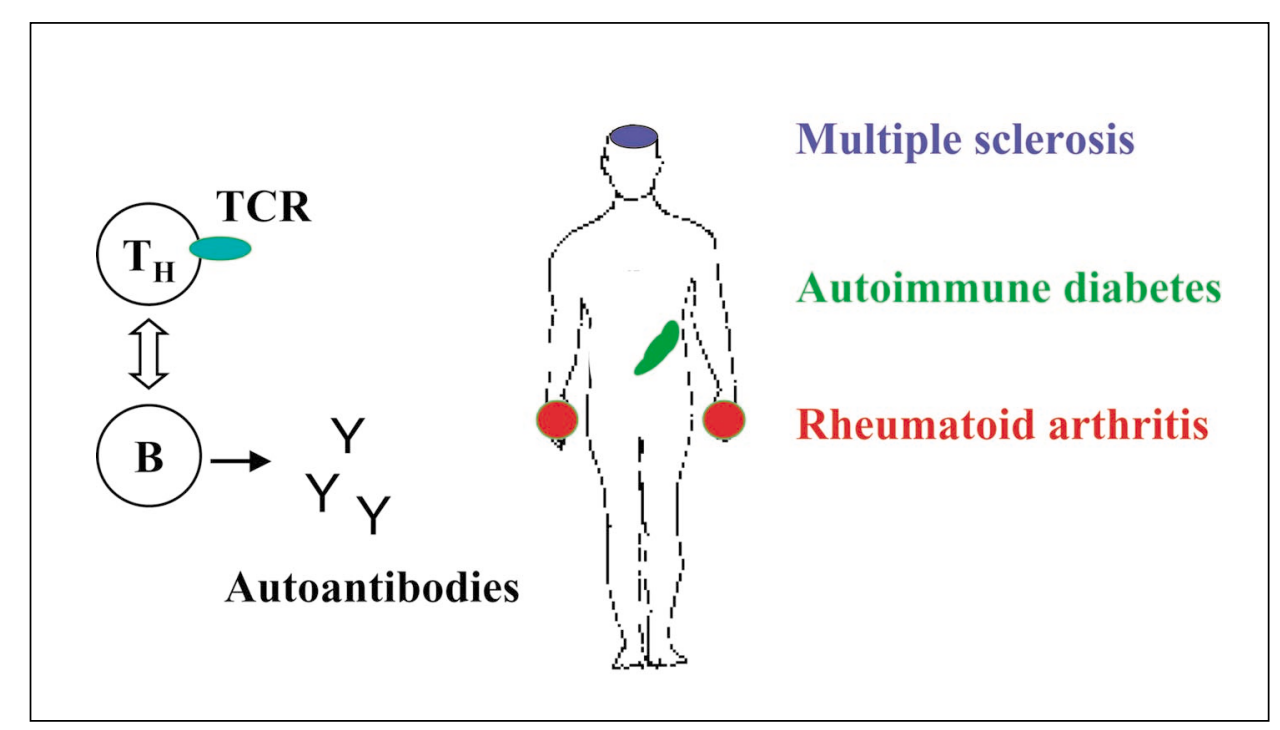

Figure 1. Autoantibodies as surrogates for the specificity of the autoimmune response in $\mathrm{T}$ cell-mediated autoimmune diseases. In T cell-mediated autoimmune diseases, including MS, IDDM, and RA, helper T cells coordinate and drive autoimmune responses to attack the target organ or tissue. Autoreactive T cells express T cell receptors (TCRs) specific for linear peptide epitopes proteolytically derived from proteins present in the organ or tissue under autoimmune attack. These autoreactive helper $\mathrm{T}$ cells reciprocally activate $\mathrm{B}$ cells that produce autoantibodies specific for epitopes on the same macromolecular complex, but typically not the exact same epitopes, as those recognized by the autoreactive $\mathrm{T}$ cells. Based on the specificity of their immunoglobulin molecules encoded by their rearranged immunoglobulin genes, B cells bind, internalize, proteolytically process, and present to $\mathrm{T}$ cells the peptides derived from macromolecular antigens, which are recognized by their immunoglobulin. This results in reciprocal activation of $\mathrm{T}$ and $\mathrm{B}$ cell responses directed against epitopes on the same macromolecular complex, enabling analysis of the specificity of the autoantibody responses as a surrogate for the specificity of $\mathrm{T}$ cell-driven autoimmune responses at the macromolecular level. 
cDNA (30,36); (ii) arrays of addressable tags, beads or nanoparticles $(8,20)$; (iii) microfluidics approaches (7); and (iv) arrays of proteins and peptides on planar surfaces $(15,18,23,34)$.

We modified the protocols of Haab et al. (9) and MacBeath and Schreiber (19) to develop planar antigen arrays to profile autoantibody responses (23). We employ widely available robotic arrayers to deposit peptides, proteins, nucleic acids, and protein complexes in ordered arrays on derivatized microscope slides. Detailed protocols are presented in our paper (22) and at http://www.stanford.edu/groups/antigenarrays.

In systemic lupus erythematosus (SLE), posttranslational modifications, including cleavage and phosphorylation, may play a critical role in the initiation of autoantibody formation and autoimmunity $(4,31)$. An important advantage of our antigen array format is the ability to detect autoantibody responses directed against posttranslational modifications within antigens.

Several groups are developing arrays of polypeptides derived from cDNA expression libraries $(3,18)$ and tissue fractions to attempt to discover the targets of autoimmune responses. In one approach, His-tagged proteins are expressed in bacteria, purified using nickel chromatography, and attached to polyvinylidene diflouride membranes for probing with autoimmune sera. This approach is being applied to study the specificity of the autoantibody response in Crohn's Disease and ulcerative colitis (34). In another approach, tissue targets of autoimmune responses are fractionated on columns, following which individual fractions are arrayed in an addressable format. Fractions containing autoimmune-specific reactivity are then further analyzed to identify the reactive polypeptide(s).

We refer readers interested in detailed descriptions of the types of proteomics technologies amenable to autoantibody profiling to several recent reviews we published on this subject $(10,25)$.

\section{LINEAR VERSUS CONFORMATIONAL B CELL EPITOPES}

In certain autoimmune diseases, including IDDM and stiff man syndrome, autoantibody responses target conformational epitopes formed through tertiary protein structures (29). For IDDM, fluid-phase assays, which detect autoantibodies specific for conformational epitopes, demonstrated greater sensitivity and specificity than nonfluid-phase assays (22). In other autoimmune diseases, including MS, the autoantibody targeting of linear epitopes has been described (35). Although debate surrounds the relevance of autoantibodies directed against linear epitopes, antigen arrays enable simultaneous profiling of autoantibodies specific for both conformational epitopes contained in recombinant proteins as well as linear eptiopes represented by peptides. Antigen array analysis of sera derived from cohorts of autoimmune patients will further clarify the relevance and clinical value of detection of autoantibodies specific for both linear and conformational epitopes.

\section{SPECIALIZED PROTEOMES TO STUDY AUTOIMMUNE DISEASES}

We are developing specialized proteome arrays that contain spectra of proteins and peptides derived from organs under autoimmune attack in different diseases. For example, connective tissue disease arrays contain a spectrum of nuclear and cellular antigens to study autoimmune rheumatic disease (23), synovial proteome arrays are composed of proteins and peptides derived from synovial joints that represent putative autoantigens in RA (10), myelin proteome arrays include myelin peptides and proteins to characterize the autoantibody response in MS (24), and islet cell proteome arrays include known pancreatic islet-derived antigens to analyze responses in IDDM.

\section{AUTOANTIBODY PROFILING FOR DIAGNOSIS AND PROGNOSTICATION}

For certain human autoimmune diseases, detection of profiles of autoantibodies provides diagnostic and prognostic utility. Detection of combinations of serum autoantibodies specific for insulin, glutamic acid decarboxylase, or the tyrosine phosphatase-like protein IA-2 are diagnostic of or prognostic for future development of IDDM (22). Detection of autoantibodies specific for nuclear antigens in combination with autoantibodies specific for the nuclear antigen Sm or DNA are highly specific for SLE (33). The diversity and epitope spreading of the autoantibody response in humans predicts subsequent development of SLE (13). It is likely that multiple additional human autoimmune diseases will have profiles of autoantibody reactivities that are diagnostic and prognostic. Antigen arrays and other proteomic technologies will facilitate identification of such profiles, including profiles that could be orders of magnitude more complicated than those currently described for IDDM and SLE.

\section{PROTEOMICS TO TAILOR TOLERIZING THERAPY}

We are using proteomically determined autoantibody profiles to guide development and selection of antigen-specific tolerizing therapies (24). Such antigen-specific therapies include conventional protein- and peptide-based tolerizing therapies, as well as genetic tolerizing vaccines. We termed the use of arraydetermined autoantibody specificities to develop and select DNA tolerizing vaccines "reverse genomics" (24). We are also applying antigen arrays to follow the response to tolerizing therapy, and we observe reductions in the diversity of autoantibody responses in animals receiving efficacious tolerizing therapy.

\section{CONCLUSION}

Proteomic profiling of autoantibody responses has the potential to determine the specificity of autoimmune responses in individuals and cohorts of patients. In an analogous fashion to the use of skin-test antigens injected intradermally in an array format on patients' backs in an allergy clinic to select desensitization therapy, proteomics technologies could be applied to tailor antigen-specific therapies to treat individuals and cohorts of patients with autoimmune disease. Proteomics technologies could also be used to select patients to receive a specific tolerizing therapy or to monitor responses to tolerizing therapy. 


\section{ACKNOWLEDGMENTS}

The authors thank B.J. Lee, W. Hueber, D. Fong, and other members of the Utz and Steinman laboratories for critical review of the manuscript. This work was supported by National Institutes of Health (NIH) Grant No. K08 AR02133 and an Arthritis Foundation Northern California Chapter Grant to W.H.R; NIH Grant Nos. K08 AI01521, U19 DK61934, U19 AI50864, an Arthritis Foundation Investigator Award, a Bio-X Grant, and a Baxter Foundation Career Development Award to P.J.U.; and NIH/National Institute of Neurological Disorders (NINDS) Grant No. 5R01NS18235 and NIH Grant No. U19 DK61934 to L.S.

\section{REFERENCES}

1.Altman, J.D., P.A. Moss, P.J. Goulder, D.H. Barouch, M.G. McHeyzerWilliams, J.I. Bell, A.J. McMichael, and M.M. Davis. 1996. Phenotypic analysis of antigen-specific T lymphocytes. Science 274:94-96.

2.Anderson, A.C., L.B. Nicholson, K.L. Legge, V. Turchin, H. Zaghouani, and V.K. Kuchroo. 2000. High frequency of autoreactive myelin proteolipid protein-specific $\mathrm{T}$ cells in the periphery of naive mice: mechanisms of selection of the self-reactive repertoire. J. Exp. Med. 191:761-770.

3.Büssow, K., D. Cahill, W. Nietfeld, D. Bancroft, E. Scherzinger, H Lehrach, and G. Walter. 1998. A method for global protein expression and antibody screening on high-density filters of an arrayed cDNA library. Nucleic Acids Res. 26:5007-5008.

4.Casciola-Rosen, L., F. Andrade, D. Ulanet, W. Wong, and A. Rosen. 1999. Cleavage by granzyme B is strongly predictive of autoantigen status. Implications for initiation of autoimmunity. J. Exp. Med. 190:815-826.

5.Celis, J.E., M. Kruhoffer, I. Gromova, C. Frederiksen, M. Ostergaard, T. Thykjaer, P. Gromov, J. Yu, et al. 2000. Gene expression profiling: monitoring transcription and translation products using DNA microarrays and proteomics. FEBS Lett. 480:2-16.

6.Chabas, D., S.E. Baranzini, D. Mitchell, C.C. Bernard, S.R. Rittling, D.T. Denhardt, R.A. Sobel, C. Lock, et al. 2001. The influence of the proinflammatory cytokine, osteopontin, on autoimmune demyelinating disease. Science 294:1731-1735.

7.Devasenathipathy, S., J.G. Santiago, and K. Takehara. 2002. Particle track ing techniques for electrokinetic microchannel flows. Anal. Chem. 74:37043713.

8.Fulton, R.J., R.L. McDade, P.L. Smith, L.J. Kienker, and J.R. Kettman, Jr. 1997. Advanced multiplexed analysis with the FlowMetrix system. Clin. Chem. 43:1749-1756.

9.Haab, B.B., M.J. Dunham, and P.O. Brown. 2001. Protein microarrays fo highly parallel detection and quantitation of specific proteins and antibodies in complex solutions. Genome Biol. 2:RESEARCH0004.0001-0004.0013.

10.Hueber, W., P.J. Utz, L. Steinman, and W.H. Robinson. 2002. Autoantibody profiling for the study and treatment of autoimmune disease. Arthritis Res. 4:290-295.

11.Jackson, R. and N. Standart. 1990. Do the poly(A) tail and 3' untranslated region control mRNA translation? Cell 62:15-24

12.Jacobson, D.L., S.J. Gange, N.R. Rose, and N.M. Graham. 1997. Epidemiology and estimated population burden of selected autoimmune diseases in the United States. Clin. Immunol. Immunopathol. 84:223-243.

13.James, J. and J. Harley. 1998. B-cell epitope spreading in autoimmunity. Immunol. Rev. 164:185-200.

14.Jiang, Z. and J. Wu. 1999. Alternative splicing and apoptosis. Proc. Soc. Exp. Biol. Med. 220:64-72.

15.Joos, T.O., M. Schrenk, P. Hopfl, K. Kroger, U. Chowdhury, D. Stoll, D. Schorner, M. Durr, et al. 2000. A microarray enzyme-linked immunosorbent assay for autoimmune diagnostics. Electrophoresis 21:2641-2650.

16.Kontoyiannis, D., M. Pasparakis, T.T. Pizarro, F. Cominelli, and G. Kollias. 1999. Impaired on/off regulation of TNF biosynthesis in mice lacking TNF AU- rich elements: implications for joint and gut-associated immunopathologies. Immunity 10:387-398.

17.Lock, C., G. Hermans, R. Pedotti, A. Brendolan, E. Schadt, H. Garren, A Langer-Gould, S. Strober, et al. 2002. Gene-microarray analysis of multiple sclerosis lesions yields new targets validated in autoimmune en- cephalomyelitis. Nat. Med. 8:500-508.

18.Lueking, A., M. Horn, H. Eickhoff, K. Büssow, H. Lehrach, and G. Walter. 1999. Protein microarrays for gene expression and antibody screening. Anal. Biochem. 270:103-111

19.MacBeath, G. and S. Schreiber. 2000. Printing proteins as microarrays for high-throughput function determination. Science 289:1760-1763.

20.Nicewarner-Pena, S.R., R.G. Freeman, B.D. Reiss, L. He, D.J. Pena, I.D. Walton, R. Cromer, C.D. Keating, and M.J. Natan. 2001. Submicrometer metallic barcodes. Science 294:137-141.

21.Pandey, A. and M. Mann. 2000. Proteomics to study genes and genomes. Nature 405:837-846.

22.Pietropaolo, M. and G.S. Eisenbarth. 2001. Autoantibodies in human diabetes. Curr. Dir. Autoimmun. 4:252-282.

23.Robinson, W.H., C. DiGennaro, W. Hueber, B.B. Haab, M. Kamachi, E.J. Dean, S. Fournel, D. Fong, et al. Autoantigen microarrays for multiplex characterization of autoantibody responses. Nat. Med. 8:295-301.

24.Robinson, W.H., H. Garren, P.J. Utz, and L. Steinman. 2002. Millennium Award. Proteomics for the development of DNA tolerizing vaccines to treat autoimmune disease. Clin. Immunol. 103:7-12.

25.Robinson, W.H., L. Steinman, and P.J. Utz. 2002. Proteomics technologies for the study of autoimmune disease. Arthritis Rheum. 46:885-893.

26.Sheets, M., C. Fox, T. Hunt, G. Vande Woude, and M. Wickens. 1994. The 3 -untranslated regions of c-mos and cyclin mRNAs stimulate translation by regulating cytoplasmic polyadenylation. Genes Dev. 8:926-938.

27.Steinman, L. 1996. A few autoreactive cells in an autoimmune infiltrate control a vast population of nonspecific cells: a tale of smart bombs and the infantry. Proc. Natl. Acad. Sci. USA 93:2253-2256.

28.Steinman, L. 1996. Multiple sclerosis: a coordinated immunological attack against myelin in the central nervous system. Cell 85:299-302.

29.Tuomi, T., M.J. Rowley, W.J. Knowles, Q.Y. Chen, T. McAnally, P.Z. Zimmet, and I.R. Mackay. 1994. Autoantigenic properties of native and denatured glutamic acid decarboxylase: evidence for a conformational epitope. Clin. Immunol. Immunopathol. 71:53-59.

30.Uetz, P., L. Giot, G. Cagney, T.A. Mansfield, R.S. Judson, J.R. Knight, D. Lockshon, V. Narayan, et al. 2000. A comprehensive analysis of protein-protein interactions in Saccaromyces cerevisiae. Nature 403:623-627.

31.Utz, P., M. Hottelet, W. van Venrooij, and P. Anderson. 1998. Association of phosphorylated SR proteins and the U1-small nuclear ribonuclear protein autoantigen complex accompanies apoptotic cell death. J. Exp. Med. 187:547-560.

32.Venter, J. M.D. Adams, E.W. Myers, P.W. Li, R.J. Mural, G.G. Sutton, H.O. Smith, M. Yandell, et al. 2001. The sequence of the human genome. Science 291:1304-1351.

33.von Mühlen, C.A. and E.M. Tan. 1995. Autoantibodies in the diagnosis of systemic rheumatic diseases. Semin. Arthritis Rheum. 24:323-358.

34.Walter, G., K. Büssow, D. Cahill, A. Lueking, and H. Lehrach. 2000. Protein arrays for gene expression and molecular interaction screening. Curr. Opin. Microbiol. 3:298-302.

35.Warren, K., I. Katz, and L. Steinman. 1995. Fine specificity of the antibody response to myelin basic protein in the central nervous system in multiple sclerosis: the minimal B cell epitope and a model of its unique features. Proc. Natl. Acad. Sci. USA 92:11061-11065.

36.Ziauddin, J. and D.M. Sabatini. 2001. Microarrays of cells expressing defined cDNAs. Nature 411:107-110.

\section{Address correspondence to:}

Dr. William H. Robinson

Stanford University School of Medicine

Beckman Center Room B-002

Stanford, CA 94305, USA

e-mail:wrobins@stanford.edu 